\section{HRCT-defined emphysema is not COPD to be treated with inhalers}

\author{
Paul Enright
}

\begin{abstract}
The COPDGene Study ${ }^{1}$ is providing important new information about the natural history of several chronic obstructive pulmonary disease (COPD) phenotypes. $^{2}$ This multicentre study enrolled a large number of current and former smokers with a wide range of spirometry results (from normal to very severe airflow obstruction), carefully characterised them during their baseline examination, and has been following them for several important outcome measures. The key phenotypes include (1) chronic airflow obstruction (CAO) defined by a low postbronchodilator forced expiratory volume in $1 \mathrm{~s}\left(\mathrm{FEV}_{1}\right) /$ forced vital capacity (FVC), (2) high-resolution CT (HRCT)-defined emphysema (low attenuation at maximal inhalation, total lung capacity), (3) hyperinflation or gas trapping on HRCT, defined as low attenuation of the lungs at low lung volume (around functional residual capacity), and (4) airway inflammation (defined by bronchial wall thickening on HRCT, also known as bronchiectasis). A comparison of two of these phenotypes-CAO versus emphysema-has been described. ${ }^{3}$ Not surprisingly, the analyses showed 'striking disagreement' between spirometric CAO (regardless of how mild airway obstruction was defined) and HRCT-defined emphysema. Only $13 \%$ of smokers with CAO had emphysema, while 39\% had air trapping.
\end{abstract}

The authors then compared the prevalence of HRCT-defined emphysema in smokers with borderline to mild CAO defined by the faulty fixed ratio versus the lower limit of the normal range (LLN) for $\mathrm{FEV}_{1} / \mathrm{FVC}$. About $7 \%$ of the adult smokers in the cohort had an $\mathrm{FEV}_{1} / \mathrm{FVC}$ below 0.70 but above the LLN (a discordant CAO classification). This 'fixed-only' subgroup of smokers had only mild CAO at worst (with a mean $\mathrm{FEV}_{1}$ of $81 \%$ predicted). The 548 participants in this subgroup were substantially older (mean age 65 vs 57), had more pack-years of smoking (due to their older age), and

Correspondence to Dr Paul Enright, Department of Medicine, The University of Arizona, PO Box 675, Mount Lemmon, Tucson, AZ 85619, USA; lungguy@gmail.com included a higher percentage of men (70\% vs 54\%) compared with the large group of smokers with entirely normal spirometry. Use of the faulty fixed ratio to define mild CAO selects older men because it does not take the natural aging of the lung into account. This subgroup of older men with borderline to mild CAO had a higher rate of HRCT-defined emphysema (4\% vs $2 \%$ ) and gas trapping $(19 \%$ vs $11 \%)$ compared with the smokers with normal spirometry.

During follow-up, the subgroup of older smokers with $\mathrm{FEV}_{1} / \mathrm{FVC}<0.70$ but above the LLN were more likely to report a mild respiratory 'exacerbation' (one every 3 years vs one every 4 years). The authors suggest that this higher rate of morbidity (along with data from other studies) provides evidence that the fixed ratio should be used to provide higher sensitivity for mild CAO (compared with the LLN), but I disagree. These episodes were defined by a positive response to the question 'Have you had a flare-up of your chest trouble in the last 12 months?' However, medical records were not reviewed to verify or classify the cause or treatment of these episodes. Some were probably due to heart failure and others due to a respiratory virus exacerbating undiagnosed asthma. Some were undoubtedly due to an exacerbation of the chronic bronchitis caused by their smoking, ${ }^{4}$ but treatment of one of these mild 'COPD exacerbations' with 10 days of prednisone and a generic antibiotic is much less expensive than daily use of a COPD inhaler (long-acting $\beta$-agonist or anticholinergic) for several years. Since their baseline lung function was near normal, very few of these episodes caused a large enough decline in lung function to cause dyspnoea, which might respond to an inhaled bronchodilator. Apparently, none of these episodes were reported to have resulted in a hospitalisation (the major COPD expense).

Home oxygen was initiated more often during follow-up in the fixed-only subgroup (25 of 548) of the COPDGene Study. However, this finding should not be used to suggest that smokers with borderline CAO have higher morbidity, since the vast majority of those with a low resting oxygen saturation were from 'mile high' Denver, Colorado (just one of the 22 study sites). ${ }^{5}$ In addition, about $13 \%$ of COPDGene Study participants with a normal resting oxygen saturation reported using supplemental oxygen continuously (obviously not prescribed on the basis of objective measurements of hypoxaemia). It is highly unlikely that progression of COPD (from borderline to very severe) was the reason for their oxygen prescriptions. HRCT-defined emphysema in this cohort was associated with dyspnoea ${ }^{6}$ but not with oxygen prescriptions.

Perhaps the industry sponsorship of COPDGene and many of the investigators influenced the authors' primary conclusion that the LLN method of defining mild CAO 'will miss a large number of patients who have significant respiratory symptoms and CT emphysema, and could potentially benefit from early detection and therapy.' However, the most recent COPD guidelines, based on extensive literature reviews, and published by four professional societies, ${ }^{7}$ does not support the widely held belief (encouraged by drug companies and the key opinion leaders who consult for them) that COPD can or must be detected when $\mathrm{CAO}$ is mild. Here are the reasons. (1) Other than smoking cessation, there is no effective treatment for mild to moderate $\mathrm{CAO}$ in smokers who do not have asthma. (2) There is certainly no treatment for the emphysema phenotype until it has progressed to the severe stage (with accompanying very severe CAO). (3) Treatment with a daily COPD inhaler in adults with an $\mathrm{FEV}_{1}$ above $60 \%$ predicted is expensive, risks serious side effects, probably reduces the motivation for the patient to stop smoking, and delays diagnostic efforts to determine the real cause of their respiratory symptoms. (4) Interventions for coronary artery disease, congestive heart failure, anxiety/depression and the metabolic syndrome (also known as COPD comorbidity) are much more effective at reducing morbidity and mortality than COPD inhaler therapy.

Because the prognosis and effective treatments differ, it is clinically important in smokers with dyspnoea to differentiate $\mathrm{CAO}$ from congestive heart failure, ${ }^{8}$ to differentiate the emphysema phenotype from the CAO phenotype, and to separate $\mathrm{CAO}$ due to poorly reversible asthma (primarily eosinophilic airway inflammation) from $\mathrm{CAO}$ due to bronchiectasis and chronic bronchitis. ${ }^{9}$ The emphysema phenotype, as defined by a low transfer factor (DLCO or KCO) or (at greater expense) by low attenuation on lung HRCT, predicts a more rapid subsequent 
loss of lung function ${ }^{10}$ and a higher death rate, ${ }^{11}$ while a normal DLCO makes asthma more likely than emphysema. Although a comparison of the $\mathrm{FEV}_{1}$ before and $10 \mathrm{~min}$ after two puffs of salbutamol is usually not helpful for differentiating asthma from COPD in an elderly adult smoker with $\mathrm{CAO}$, the longer-term $\mathrm{FEV}_{1}$ and symptom response to a high daily dose of an inhaled corticosteroid taken for 1-3 months will reveal the more fortunate patients with 'hidden asthma'. ${ }^{12}$

In summary, the COPDGene Study and other investigators have successfully defined several phenotypes in adult smokers, many of which overlap with other COPD phenotypes and comorbid conditions. The intervention that has been proven to reduce morbidity and mortality is smoking cessation, so the majority of effort and expense should be directed towards this difficult process. There is no evidence that the cost/benefit ratio of daily COPD inhalers is favourable for patients with CAO unless their $\mathrm{FEV}_{1}$ is below $60 \%$ predicted. No inhaler will help those with HRCT-defined emphysema and mild CAO. Therefore, the only imperative that I see to detect COPD early is to 'sell sickness' and thus enhance the billion dollar (pound or Euro) annual profits of companies that sell COPD inhalers. ${ }^{13}$
Competing interests None.

Provenance and peer review Commissioned; internally peer reviewed.

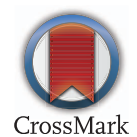

To cite Enright P. Thorax 2014;69:401-402.

Published Online First 6 January 2014

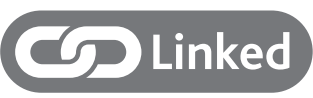

http://dx.doi.org/10.1136/thoraxjnl-2012-202810

Thorax 2014;69:401-402.

doi:10.1136/thoraxjnl-2013-203497

\section{REFERENCES}

1 Regan EA, Hokanson JE, Murphy JR, et al. Genetic epidemiology of COPD (COPDGene) study design. COPD 2010;7:32-43

2 Han MK, Agusti A, Calverley PM, et al. COPD phenotypes: the future of COPD. Am J Respir Crit Care Med 2010;182:598-604.

3 Bhatt SP, Sieren JC, Dransfield MT, et al. Comparison of spirometric thresholds in diagnosing smoking related airflow obstruction. Thorax 2014;69:410-5.

4 Kim V, Han LK, Vance GB, et al. The chronic bronchitis phenotype of COPD: an analysis of the COPDGene study. Chest 2011;140:626-33.

5 Kim DK, Jacobson FL, Washko GR, et al. Clinical and radiographic correlates of hypoxemia and oxygen therapy in the COPDGene study. Respir Med 2011;105:1211-21.

6 Diaz AA, Valim C, Yamashiro T, et al. Airway count and emphysema assessed by chest $\mathrm{CT}$ imaging predicts clinical outcome in smokers. Chest 2010;138:880-7.

7 Qaseem A, Wilt TJ, Weinberger SE, et al. Diagnosis and management of stable COPD: a clinical practice guideline update from the American College of Physicians, American College of Chest Physicians, American Thoracic Society, and European Respiratory Society. Ann Intern Med 2011;155:179-91.

8 Hawkins NM, Petrie MC, Jhund PS, et al. Heart failure and chronic obstructive pulmonary disease: diagnostic pitfalls and epidemiology. Eur J Heart Failure 2009;11:130-9.

9 Al-Kassimi FA, Alhamad EH. A challenge to the seven widely believed concepts in COPD. Inter J COPD 2013;8:21-30.

10 Nishimura M, Makita H, Nagai K, et al. Annual change in pulmonary function and clinical phenotype on COPD. Am J Respir Crit Care Med 2012;185:44-52.

11 Al-Kassimi FA, Abba AA, Al-Hajjaj MS, et al. Asthma masquerading as COPD: a study of smokers fulfilling the GOLD definition of COPD. Respiration 2011;82:19-27.

12 Leigh R, Pizzichini MM, Morris MM, et al. Stable COPD: predicting benefit from high-dose inhaled corticosteroid treatment. Eur Respir J 2006;27:964-71.

13 Weber LJ. Profits before people? Ethical standards and the marketing of prescription drugs. Bloomington, IN: Indiana University Press, 2006. 